\title{
Loss of a paternal chromosome causes developmental anomalies among Drosophila hybrids
}

\author{
JOHN M. BRAVERMAN, BEATRIZ GOÑI* \& H. ALLEN ORR \\ Center for Population Biology and "Department of Genetics, University of California, Davis, CA 95616, U.S.A.
}

\begin{abstract}
Hybrids between Drosophila virilis and $D$. lummei suffer from developmental anomalies. Previous reports also suggest that these hybrids lose the $D$. lummei sixth chromosome early in development. Genetic and cytological data presented here confirm the loss of the microchromosome from both the soma and the germ-line of these hybrids and provide strong evidence that this loss causes the hybrid developmental anomalies.
\end{abstract}

Keywords: chromosome loss, Drosophila lummei, Drosophila virilis, hybrid inviability, morphological anomalies, speciation.

\section{Introduction}

Hybrid sterility and inviability are important forms of reproductive isolation in nature but their physiological bases are usually unknown. One exception is the syndrome of anomalies in hybrids between Drosophila virilis and D. lummei, which includes such deleterious traits as reduced eyes, unequal wing lengths, twisted abdomens, missing or reduced thoracic bristles, incomplete sclerotization of the abdomen, and uninflated, broken, or incomplete wing veins (Sokolov, 1948, 1959). There is some evidence to suggest that these anomalies result from somatic loss of the $D$. lummei microchromosome (the 'dot' or sixth chromosome) from hybrids (Orr, 1990). Individuals that lose their microchromosomes, for example, show far more anomalies than those that do not. Although Heikkinen (1991) also observed this correlation, she recently concluded that the anomalies do not result from microchromosome loss.

This note has two purposes: (i) to confirm that the $D$. lummei dot chromosome is, in fact, lost among $D$. virilis $-D$. lummei hybrids, and (ii) to test directly whether microchromosome loss causes the hybrid anomalies.

Previous evidence that hybrids lose the microchromosome has been indirect, largely based on the behaviour of the microchromosomal marker glossy $(\mathrm{gl}$ $6-1.0)$ among hybrids. Although glossy is recessive, $F_{1}$ hybrids that have $D$. virilis mothers are often mosaic or completely glossy (Sokolov, 1948, 1959; Evgen'ev \&
Sidorova, 1976; Orr 1990; Heikkinen, 1991). These observations have been interpreted as the result of a loss of the wild-type $D$. lummei chromosome from the hybrid embryo.

However, other explanations for the appearance of glossy are possible. For instance, hybrids might appear glossy if the normally-recessive $g l$ allele acts dominantly among hybrids. Such reversals of dominance in species hybrids are well-known (Muller, 1942). Alternatively, the heterochromatic nature of the dot chromosome (Miklos, et al., 1988; Ashburner, 1989, chapter 23) may predispose it to position-effect variegation, and this may result in glossy mosaicism.

Cytological evidence for loss of the $D$. lummei dot chromosome is also not strong. Evgen'ev \& Sidorova (1976) claim that this chromosome is usually missing from salivary gland preparations of hybrids. However, the tiny dot chromosome is often entangled with the chromocentre and is difficult to see. More convincing evidence of chromosome loss would come from mitotic cells, where the number of microchromosomes can be scored more confidently.

The genetic and cytological data presented here demonstrate that the $D$. lummei dot chromosome is indeed lost from somatic tissues of hybrids. The temperature-sensitivity of chromosome loss, whether such loss occurs in the germ-line, and whether hybrids also lose other chromosomes, are also investigated. Finally, our data provide strong evidence that this loss causes the syndrome of developmental anomalies seen among these hybrids. 
Table 1 Glossy phenotypes of $F_{1}$ hybrids. Mosaics are classified as 1/2-eye glossy (Class 2), 1-eye glossy (Class 3), or 1-1/2 eye glossy (Class 4)

\begin{tabular}{lllrrrrr}
\hline \multirow{2}{*}{ Genotype } & & Temperature & $\begin{array}{c}\text { Both-eyes } \\
\text { wild-type }\end{array}$ & $\begin{array}{r}\text { Mosiac } \\
\text { Class 2 }\end{array}$ & $\begin{array}{r}\text { Mosiac } \\
\text { Class 3 }\end{array}$ & $\begin{array}{l}\text { Mosiac } \\
\text { Class 4 }\end{array}$ & \multicolumn{2}{l}{$\begin{array}{l}\text { Both-eyes } \\
\text { glossy }\end{array}$} & Total \\
\hline VL & $22^{\circ} \mathrm{C}$ & 224 & 101 & 262 & 92 & 247 & 926 \\
LV & $22^{\circ} \mathrm{C}$ & 239 & 0 & 0 & 0 & 0 & 239 \\
VL & $18^{\circ} \mathrm{C}$ & 0 & 0 & 7 & 4 & 876 & 887 \\
\hline
\end{tabular}

\section{Materials and methods}

The fly stocks used were $D$. virilis peach; glossy [pe: 5-203; gl: 6-1.0; map positions from Alexander (1976)] and D. lummei Finland. These stocks were obtained from the Bowling Green Species Stock Center. Unless otherwise indicated, all crosses were performed at $22^{\circ} \mathrm{C}$ as described by $\operatorname{Orr}(1990)$.

We examined the karyotypes of $D$. virilis, $D$. lummei and $D$. virilis $-D$. lummei hybrids in somatic and germ cells. Mitotic cells from cerebral ganglia of third instar larvae or prepupae were karyotyped. Gonial and meiotic cell divisions from the testes of newly emerged adults were also karyotyped. Cytological preparations were made following the air-drying method of Imai $e t$ al. (1988), without colchicine treatment. This method produces C-banded metaphase karyotypes. All cytological preparations were made from single flies and data was collected only from those cells in which the full chromosome complement was clearly visible.

To abbreviate the description of crosses, the following notation is used: $\mathrm{V}=D$. virilis and $\mathrm{L}=D$. lumme $i$; in hybrids and backcrosses the maternal parent is always listed first. Thus (VL)L represents the backcross of $F_{1}$ hybrid females that have $D$. virilis mothers, to $D$. lummei males.

\section{Results}

Is the D. lummei microchromosome lost from somatic tissues of hybrids? As expected (Sokolov, 1948, 1959; Evgen'ev \& Sidorova, 1976; Orr, 1990), $F_{1}$ hybrids from the cross $D$. virilis pe;gl $\times D$. lummei are usually glossy or glossy mosaics (Table 1, line 1). Many of these flies show a weak Minute phenotype [the dot chromosome of most, if not all, Drosophila species carries a Minute locus (Ashburner, 1989, chapter 23)]. The reciprocal cross, however, does not produce glossy offspring (line 2). Glossy phenotypes among $F_{1}(V L)$ hybrids are much more common at lower temperatures (line 3), as previously reported (Evgen'ev \& Sidorova,
Table 2 Cytological observations in somatic (cerebral ganglia) cells in D. virilis, D. lummei and $\mathrm{F}_{1}(\mathrm{VL})$ reared at $18^{\circ} \mathrm{C}$

\begin{tabular}{lcll}
\hline Genotype & Individuals & Mitotic cells & Haplo-6 cells \\
\hline $\begin{array}{l}\text { D. virilis pe;gl } \\
\quad\end{array}$ & & & \\
$\quad$ Male & 11 & 53 & 0 \\
$\quad$ Female & 4 & 12 & 0 \\
$\begin{array}{l}\text { D. lummei } \\
\quad \text { Male }\end{array}$ & 12 & 59 & 0 \\
$\quad$ Female & 4 & 17 & 0 \\
F, VL $)$ & & & \\
$\quad$ Male & 9 & 43 & 43 \\
Female & 7 & 34 & 34 \\
\hline
\end{tabular}

1976). The important question is whether this glossy phenotype reflects somatic microchromosomes loss or whether it reflects one of the alternative explanations described in the Introduction.

To test the chromosome loss hypothesis, the karyotypes of cerebral ganglia cells from both pure species and hybrid larvae were examined (all flies raised at $18^{\circ} \mathrm{C}$ ). The data are shown in Table 2 and are illustrated in Fig. $1 \mathrm{a}-\mathrm{d}$. The chromosome complement of the two species consists of five pairs of rods and a pair of dot (or sixth) chromosomes (Throckmorton, 1982). All pure species individuals are diplo- 6 , while all hybrid individuals are haplo-6. Thus microchromosome loss does frequently occur among $F_{1}(V L)$ hybrids, and the glossy phenotype can be used as a convenient indicator of this chromosome loss.

Is the microchromosome lost from the germ-line? There has been some confusion about whether chromosome loss also occurs in the germ-line of hybrids. Evgen'ev \& Sidorova (1976), who constructed a special stock carrying the $D$. lummei dot chromosome in an otherwise $D$. virilis genome, reported that germ-line loss within this stock either does not occur or is very rare. 


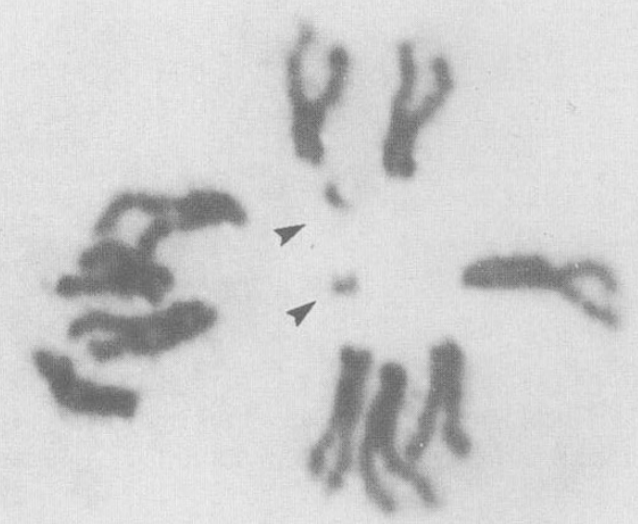

(a)

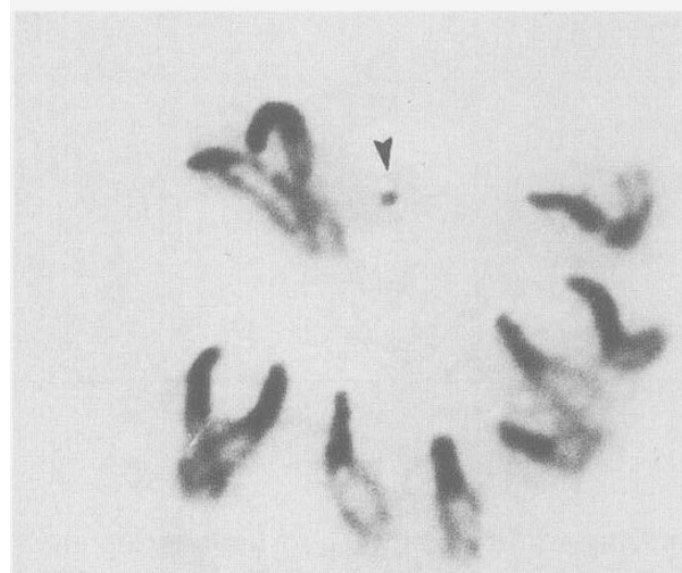

(c)

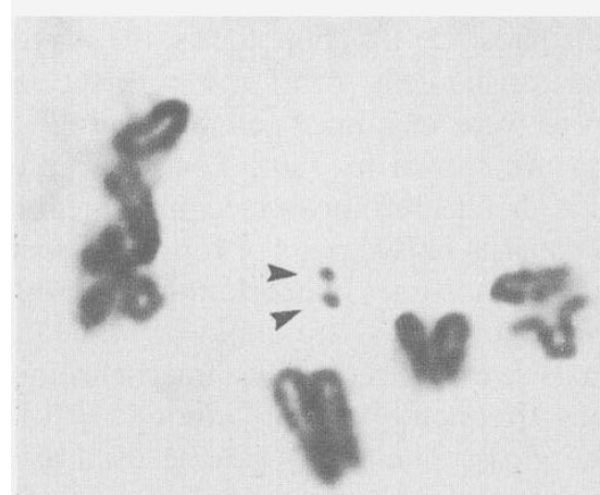

(e) (b)
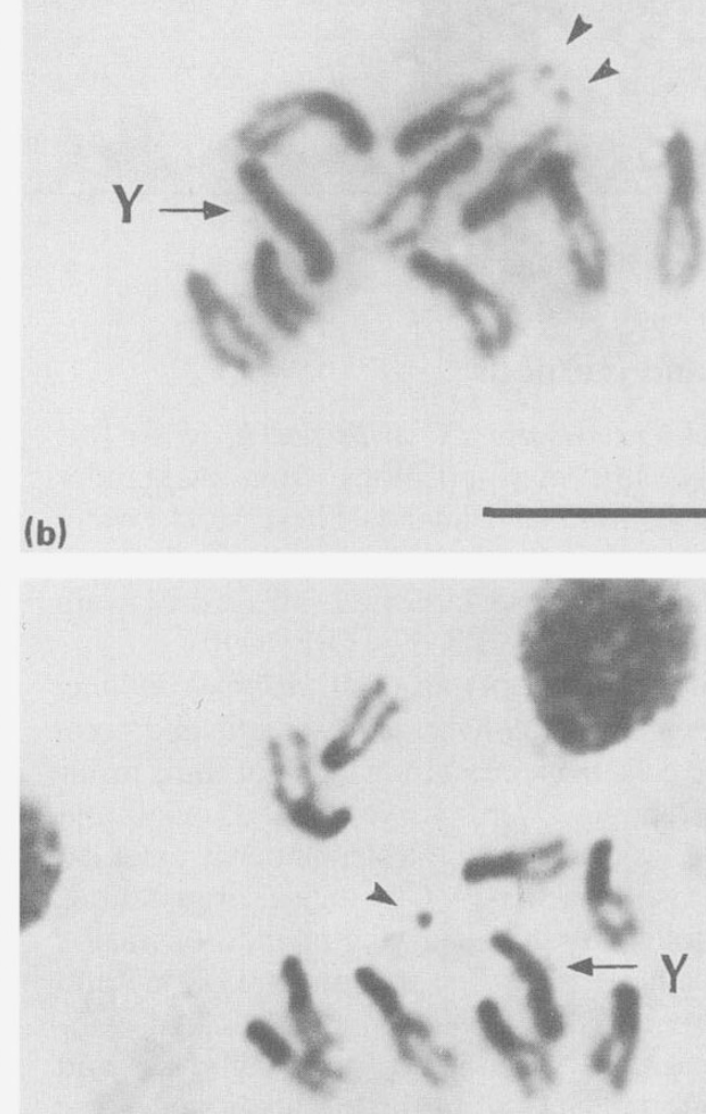

(d)

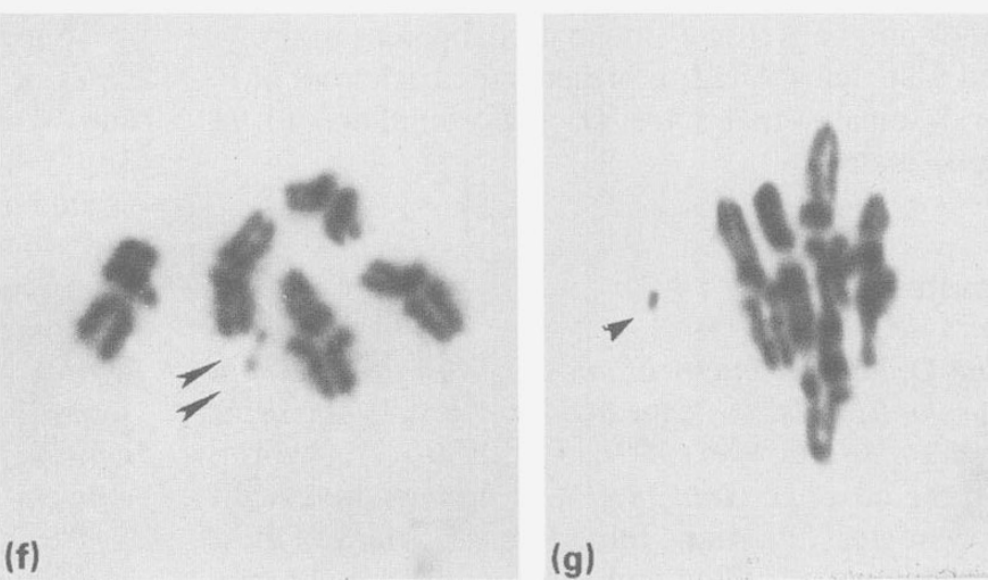

Fig. 1 Sample micrographs of D. virilis, D. lummei, and $\mathrm{F}_{1}(\mathrm{VL})$ hybrid chromosomes in C-band mitotic metaphase $(\mathrm{a}-\mathrm{d})$ and meiotic metaphase I and anaphase I (e-g): (a) D. virilis female, (b) D. lummei male, (c) $\mathrm{F}_{1}(\mathrm{VL})$ female, (d) $\mathrm{F}_{1}(\mathrm{VL})$ male, (e) D. virilis male, (f) D. lummei male and $(\mathrm{g}) \mathrm{F}_{1}(\mathrm{VL})$ male. The dot chromosomes are indicated by arrow heads. The heterochromatic Y-chromosome is indicated in (b) and (d). Bar $=10 \mu \mathrm{m}$. 
This does not necessarily mean, however, that germline loss does not occur among $F_{1}(V L)$ hybrids.

The karyotypes of gonial and meiotic cells from the testes of both pure species individuals and hybrid flies were examined. The microchromosome was always missing from the germ-line of hybrids (Table 3). Sample micrographs appear in Fig. $1 \mathrm{e}-\mathrm{g}$.

To verify that the $D$. lummei, and not the $D$. virilis, microchromosome is missing from germ cells, individual 'whole-eye glossy' $\mathrm{F}_{1}(\mathrm{VL})$ males - which lack a $D$. lummei microchromosome in most or all of their somatic tissues - were backcrossed to $D$. virilis pe;gl females. If a male has also lost the D. lummei dot chromosome from its germ-line, then all of its progeny should be gl (progeny were reared at $22^{\circ} \mathrm{C}$ to minimize zygotic chromosome loss). The absence of $\mathrm{gl}^{+}$progeny could, however, have another cause: the $D$. lummei microchromosome on a largely $D$. virilis genetic background may simply result in inviability. To control for this possibility, individual $\mathrm{gl}^{+} \mathrm{F}_{1}(\mathrm{VL})$ males which have not lost the $D$. lummei dot chromosome from their soma - were backcrossed to $D$. virilis pe;gl females. These males will produce $\mathrm{gl}^{+}$progeny unless the $D$. lummei sixth on a $D$. virilis background causes inviability. In the experimental cross (glossy fathers), only three of 15 hybrid males produced any $\mathrm{gl}^{+}$progeny. In the control cross (wild-type fathers), 26 of 32 males produced $\mathrm{gl}^{+}$progeny $\left(\chi^{2}=16.2\right.$, d.f. $=1$, $P<0.0001$; data included only if parental male produced seven or more offspring). Thus the absence of the D. lummei dot among the progeny of experimental males is not an artifact of hybrid inviability. Instead, the $D$. lummei dot chromosome is often missing from the germ cells of $F_{1}(V L)$ males. This may well reflect loss of the chromosome before the germ-line is set aside in early embryogenesis [in $D$. melanogaster, pole cells form in the ninth division (Ashburner, 1989, p. 170)].

Are other chromosomes lost from hybrids? Evgen'ev \& Sidorova (1976) suggest that there is nothing special about the dot chromosome: loss of other $D$. lummei chromosomes from hybrids might be common. They argue, however, that the inviability of embryos that lack a major chromosome would prevent easy detection of such chromosome loss. Loss of the dot chromosome, on the other hand, is easily detected among adult hybrids because haplo-dot chromosome flies are viable in Drosophila (Lindsley \& Grell, 1968).

As a partial test of this hypothesis, we scored the presence of the $\mathrm{Y}$ chromosome among hybrid males. This is the only other chromosome whose loss can be tolerated in Drosophila [XO flies are viable in $D$. virilis (Alexander, 1976)]. Cytological data from $18 \mathrm{~F}_{1}(\mathrm{VL})$ males reared at $18^{\circ} \mathrm{C}$ revealed no other chromosome aneuploidy; the $\mathrm{Y}$ chromosome was present in all cases (Fig. 1d). This result demonstrates that all D. lummei chromosomes do not have equal chances of loss, and it suggests that the microchromosome may be particularly prone to loss. It remains possible, however, that the $\mathrm{Y}$ chromosome is particularly resistant to loss.

Does microchromosome loss cause the developmental anomalies among hybrids? To determine if microchromosome loss causes the hybrid anomalies, we produced two hybrid genotypes which differed, on average, only at the microchromosome: the first genotype inherited a $D$. lummei dot (which could then be lost early in development) and a $D$. virilis dot, while the second genotype inherited only $D$. virilis dot chromosomes (which are not lost). If hybrid anomalies result from loss of the $D$. lummei dot, the first genotype should frequently show anomalies, while the second should never (or very rarely) show anomalies.

The first genotype was produced by backcrossing $\mathrm{pe}^{+} ; \mathrm{gl}^{+} \mathrm{F}_{2}(\mathrm{LV})$ males, which carry at least one $D$. lummei microchromosome, to $D$. virilis pe;gl females. Zygotes resulting from this cross were $g l^{+} / g l$ or $g l / g l$. The second genotype was produced by backcrossing $\mathrm{pe}^{+} ; \mathrm{gl} \mathrm{F}_{2}(\mathrm{LV})$ males, which carry two $D$. virilis dot chromosomes, to $D$. virilis pe;gl females. All progeny from this cross were $\mathrm{gl} / \mathrm{gl}$. Because all $\mathrm{F}_{2}$ males were derived from an initial LV (not VL) cross, these $F_{2}$ males have experienced almost no chromosome loss and are almost all diplo- 6 . The genetic background was

Table 3 Cytological observations in germ cells in $D$. virilis, D. lummei, and $\mathrm{F}_{1}(\mathrm{VL})$ reared at $18^{\circ} \mathrm{C}$

\begin{tabular}{lllll}
\hline Genotype & Males & Gonial cells & Meotic cells & Haplo-6 cells \\
\hline D. virilis pe;gl & 10 & 14 & 13 & $0^{*}$ \\
D. lummei & 12 & 27 & 13 & 0 \\
$\mathrm{~F}_{1}(\mathrm{VL}) \mathrm{pe}^{+} ; \mathrm{gl}$ & 16 & 27 & 13 & 40 \\
\hline
\end{tabular}

*One gonial mitotic cell had $2 n=14$ (including one pair of dot chromosomes and a $\mathrm{Y}$ chromosome), instead of the usual $2 n=12$. 
Table 4 Test of dependence of hybrid anomalies on microchromosomal genotype. 'Eye', 'wing' and 'vein' refer to types of hybrid anomalies. The total per cent anomalous is a weighted average. See text for details

\begin{tabular}{llrrrrrr}
\hline $\begin{array}{l}\text { Parental } \\
\text { male }\end{array}$ & Offspring & Normal & Eye & Wing & Vein & Total & $\begin{array}{l}\text { Per cent } \\
\text { anomalous }\end{array}$ \\
\hline $\mathrm{pe}^{+} ; \mathrm{gl}^{+}$ & gl & 509 & 11 & 1 & 0 & 521 & 2.30 \\
& $\mathrm{gl}^{+}$ & 38 & 0 & 18 & 4 & 60 & 36.67 \\
& gl-mosaic & 64 & 27 & 20 & 6 & 117 & 45.30 \\
& Total & 611 & 38 & 39 & 10 & 698 & 12.46 \\
$\mathrm{pe}^{+} ; \mathrm{gl}$ & $\mathrm{gl}$ & 1068 & 0 & 0 & 3 & 1071 & 0.28 \\
\hline
\end{tabular}

partly controlled by using only $\mathrm{F}_{2}$ males that carried at least one $D$. lummei fifth chromosome (i.e. all males were $\left.\mathrm{pe}^{+}\right)$. The frequency and type of anomalies was scored for each eye phenotype produced in each cross.

Table 4 reports the results from each cross. In the first cross, over 12 per cent of all progeny showed an anomaly. In the second cross (where hybrids do not inherit a $D$. lummei dot) only 0.28 per cent showed any anomaly $\left(\chi^{2}\right.$ pooling anomalous types $=129.9$, d.f. $=2$, $P<0.0001)$. Out of over 1,000 hybrids scored, no eye or wing-length anomalies ever appeared in this second cross (three subtle wing venation variants were seen), although these anomalies were common among the progeny of the first cross. Thus, among diplo- 6 zygotes, appearance of these dramatic anomalies requires inheritance of the $D$. lummei microchromosome. Subtle wing venation variations may, however, have a different or additional genetic cause.

Table 4 provides further evidence of microchromosome involvement in the anomalies. Among the progeny of the first cross, about 40 per cent of the $\mathrm{gl}^{+}$and gl-mosaic flies (which definitely inherited a $D$. lummei dot) showed anomalies, while only about 2 per cent of the gl flies (most of which did not inherit a D. lummei dot $)$ showed anomalies $\left(\chi^{2}=194.4, \quad\right.$ d.f. $=2$, $P<0.0001)$. The low frequency of anomalies among $\mathrm{gl}$ flies presumably reflects the small fraction of gl flies which are $g l / 0$.

An additional detail from these crosses indicates that microchromosome loss causes the hybrid anomalies. While gl-mosaic flies show frequent wing-length, wing venation and eye anomalies, $\mathrm{gl}^{+}$flies show no eye anomalies, although they frequently exhibit other anomalies (Table 4). These two phenotypes differ in only one respect: although they begin with identical zygotic genotypes, gl-mosaics have lost the $D$. lummei dot from patches of eye tissue, while $\mathrm{gl}^{+}$flies have not (they may, however, have lost the dot from patches of other tissues). The fact that flies that have lost the dot from eye tissue show eye anomalies, while flies that have not lost the dot from eye tissue do not show eye anomalies, represents strong evidence that loss of the D. lummei microchromosome is the cause of the hybrid anomalies.

\section{Discussion}

The $D$. lummei microchromosome is frequently lost from both the soma and the germ-line of $D$. virilis- $D$. lummei hybrids. The results presented here also confirm that this chromosome loss is temperature-sensitive and suggest that other D. lummei chromosomes (at least the $\mathrm{Y}$ chromosome) are not lost as frequently from hybrids.

These results also provide strong evidence that loss of the D. lummei dot chromosome causes the developmental anomalies observed among these hybrids. The most direct evidence is based on a comparison of two hybrid genotypes that differ only in the species origin of the dot chromosomes. Although hybrids that inherit a D. lummei microchromosome often suffer from developmental anomalies, those which inherit only $D$. virilis microchromosomes show almost no anomalies. In addition, while backcross hybrids showing chromosome loss in eye tissues often suffer eye anomalies, hybrids not showing chromosome loss from the eye do not suffer eye anomalies (also see Orr, 1990). This pattern is evidence that the appearance of hybrid anomalies involves loss of the D. lummei microchromosome.

Heikkinen (1991), however, recently concluded that loss of the $D$. lummei microchromosome does not cause the hybrid anomalies. Her conclusion is primarily based on the claim that chromosome loss and the eye anomalies have different genetic bases. Specifically, Heikkinen found that the eye anomaly depends primarily on maternal genotype at chromosomes 2 and 5 (with 2 having the largest effect, see her fig. 3 ). 
Mitrofanov \& Sidorova (1979), however, found that microchromosome loss involves a large effect of maternal chromosome 2 and smaller effects of all other major autosomes (all results from $25^{\circ} \mathrm{C}$ ). Further comparison of Heikkinen's data with Mitrofanov \& Sidorova's (1979) caused Heikkinen to conclude that chromosome 4 has a smaller effect on the hybrid anomalies than on chromosome loss, while chromosome 5 shows the reverse pattern.

Although these data do suggest some subtle differences between the genetics of hybrid chromosome loss and hybrid anomalies, note that these studies were performed many years apart using different strains in different laboratories. Considering the uncertainty in such an uncontrolled comparison, the similarities in genetic basis seem more significant than the differences mentioned above. Both microchromosome loss and the hybrid anomalies occur non-reciprocally $(D$. virilis mothers in both cases). Both involve maternal effects. In both cases, the maternally acting genes from $D$. virilis act recessively. Finally, in both cases, maternal chromosome 2 has the largest effect.

In addition, Heikkinen's claim that the two characters have different genetic bases seems contradicted by her own data. Heikkinen (1991, table 3) found, as did $\operatorname{Orr}(1990)$, that there is an extremely stong correlation between those $F_{1}(V L)$ hybrids that show chromosome loss and those that show the anomalies. Given that all $F_{1}$ hybrids have identical zygotic genotypes (i.e. a haploid complement of chromosomes from each species) and identical maternal genotypes, the two characters cannot have separate genetic bases. If these characters had different genetic bases, it is unclear why flies of identical zygotic and maternal genotype would show either both characters or neither.

Heikkinen's direct test of the effect of the microchromosome on the hybrid anomalies also has some problems. In this test, Heikkinen compared the frequency of anomalies among the progeny of $F_{1}(\mathrm{LV})$ versus $\mathrm{F}_{1}(\mathrm{VL})$ males backcrossed to $D$. virilis females. Under the chromosome loss hypothesis, the progeny of $F_{1}(L V)$ males will often inherit (and subsequently lose) a D. lummei dot, and so will show anomalies. However, because many of the reciprocal $F_{1}(V L)$ males have already lost their dot chromosome, Heikkinen (p. 365) argued that their progeny will not inherit a $D$. lummei dot and so should not show anomalies. Because the progeny of both types of male show roughly equal frequencies of anomalies. Heikkinen ( $p$. $357)$ concludes that 'the role of the sixth chromosome (in producing the eye anomaly) is not decisive (p. 361).

However, the prediction that the progeny of $F_{j}(V L)$ males which have already lost their $D$. lummei dot, should not show anomalies assumes that a hybrid zygote must inherit and then lose a $D$. lummei dot in order to show anomalies. It is not clear, however, why a hybrid, which is haplo- 6 from the moment of fertilization (i.e. one resulting from a nullo- 6 sperm from a $F_{1}(V L)$ male) would not also show anomalies. If so, then one would expect the progeny of both $F_{1}(V L)$ and $\mathrm{F}_{1}(\mathrm{LV})$ males to show anomalies, as observed. In any case, the present data clearly show that the sixth chromosome does, in fact, play a decisive role in the hybrid anomalies.

Although chromosome loss is well-documented among plant hybrids [e.g. in barley, see Bothmer et al. (1991) and Bennett et al. (1976)], it is unknown how common it is among animals. It remains possible that chromosome loss is fairly common among animal hybrids and that it is a frequent cause of hybrid inviability. However, because early loss of a major chromosome would presumably be embryonic lethal, hybrid chromosome loss would usually go undetected (we know of no case other than $D$. virilis-D. lummei in which chromosome loss has been looked for among hybrid embryos). Whether chromosome loss among $D$. virilis $-D$. lummei hybrids represents a rare, isolated incident or some more common phenomenon will remain unknown until additional animal hybridizations are studied.

\section{Acknowledgements}

We thank J. Coyne, C. Langley, M. Orr, and T. Prout for helpful discussion and comments. This work was supported by a Sloan Post Doctoral Fellowship to $\mathrm{HAO}$.

\section{References}

aleXander, M. L. 1976. The genetics of Drosophila virilis. In: Ashburner, M. and Novitski, E. (eds) The Genetics and Biology of Drosophila, Vol. 1c, Academic Press, London. ASHBURner, M. 1989. Drosophila: a Laboratory Handbook. Cold Spring Harbor Laboratory Press, New York.

BENNETT, M. D., FINCH, R. A. AND BARCLAY, I. R. 1976. The time rate and mechanism of chromosome elimination in Hordeum by hybrids. Chromosoma, 54, 175-200.

BOTHMER, R. VON, SALOMON, B. AND LINDE-LAURSEN, I. 1991. Cytogenetics in hybrids of Hordeum jubatum and H. tetraploidium with cultivated barley $(H$. vulgare 1.$)$. Hereditas, 114, 41-46.

EVGEN'EV, M. B. AND SIDOROVA, N. v. 1976. Genetic regulation of chromosome behavior in interspecific hybrids of Drosophila. Theor. Appl. Gen., 48, 55-61.

HEIKKINEN, E. 1991. Wrinkling of the eye in hybrids between Drosophila virilis and Drosophila lummei is caused by interaction of maternal and zygotic genes. Heredity, 66, $357-365$. 
IMAI, H. T., TAYLOR, R. W., CROSLAND, M. W. J. AND CROZIER, R. H. 1988. Modes of spontaneous chromosomal mutation and karyotype evolution in ants with reference to the minimum interaction hypothesis. Jpn J. Genet., 63, 159-185.

LINDSLEY, D. L. AND GRELL, E. H. 1968. Genetic Variations of Drosophila melanogaster. Carnegie Inst. Wash. Publ, 627. Washington, DC.

MIKLOS, G. L. G., YAMAMOTO, M. -T., DAVIES, J. AND PIRROTTA, V. 1988. Microcloning reveals a high frequency of repetitive sequences characteristic of chromosome 4 and the $B$ heterochromatin of Drosophila melanogaster. Proc. Natl. Acad. Sci., U.S.A., 85, 2051-2055.

Mitrofanov, V. G. AND SIDOROVA, N. V. 1979. Phenogenetic analysis of chromosome behavior during mitosis in female Drosophila virilis X male Drosophila lummei hybrids. Soviet Genetics, 15, 807 811.

MULLER, H. J. 1942. Recessive genes causing interspecific sterility and other disharmonies between Drosophila melanogaster and simulans. Genetics, 27, 157.

ORR, H. A. 1990. Developmental anomalies in Drosophila hybrids are apparently caused by loss of microchromosome. Heredity, 64, 255-262.

soKolov, N. N. 1948. Elimination of chromosomes in interspecific hybrids of Drosophila and the problems of distant hybrids. Dokladi Academii Nauk, 59, 163-166. (In Russian).

sokolov, N. N. 1959. The interaction of nucleus and cytoplasm in distant hybrids. Moscow: Izdatelstvo Academii Nauk, 1-148 (In Russian).

THROCKMORTON, L. H. 1982. The virilis species group. In: Ashburner, M., Carson, H. L. and Thompson, J. N. Jr. (eds) The Genetics and Biology of Drosophila. Vol. 3b, Academic Press, London, pp. 227-296. 